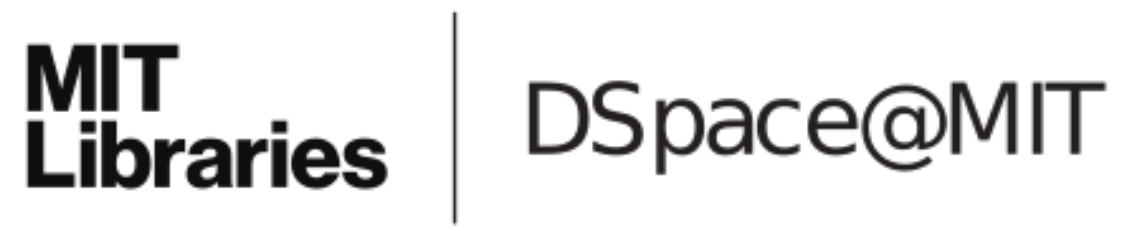

MIT Open Access Articles

Multi-Coset Sparse Imaging Arrays

The MIT Faculty has made this article openly available. Please share how this access benefits you. Your story matters.

Citation: Krieger, James D., Yuval Kochman, and Gregory W. Wornell. "Multi-Coset Sparse Imaging Arrays." IEEE Trans. Antennas Propagat. 62, no. 4 (n.d.): 1701-1715.

As Published: http://dx.doi.org/10.1109/tap.2014.2299819

Publisher: Institute of Electrical and Electronics Engineers (IEEE)

Persistent URL: http://hdl.handle.net/1721.1/91092

Version: Original manuscript: author's manuscript prior to formal peer review

Terms of use: Creative Commons Attribution-Noncommercial-Share Alike 


\title{
Multi-Coset Sparse Imaging Arrays
}

\author{
James D. Krieger, Yuval Kochman Member, IEEE, Gregory W. Wornell Fellow, IEEE
}

\begin{abstract}
We develop an efficient structured sparse antenna array architecture for coherent imaging of sparse but otherwise unknown scenes. In this architecture, the array elements are configured in a periodic nonuniform pattern that can be viewed as the superposition of multiple sparse uniform arrays. For this architecture, we develop an efficient pattern design procedure using co-array analysis. Moreover, we describe robust and efficient algorithms implementing the associated array processing needed for this architecture, which comprise scene support recovery, followed by image reconstruction. Finally, we develop a practical method for detecting reconstruction failures when the scene density exceeds the level for which the array was designed to accommodate, so that false images are not produced. Finally, as a demonstration of its viability, the architecture is used to reconstruct a simulated natural scene.
\end{abstract}

Index Terms-Phased-array antennas, sparse arrays, compressed sensing, MUSIC algorithm, millimeter-wave imaging.

\section{INTRODUCTION}

$\mathbf{R}$ ECENT advances seen in millimeter-wave technology, including the advent of terahertz CMOS circuits, have the potential to enable, for the first time, a host of low-cost imaging and "personal radar" applications. Indeed, at these higher frequencies, typical resolution requirements can be met with comparatively compact arrays, which are especially attractive for applications requiring some degree of mobility. Moreover, the arrays can be implemented with inexpensive integrated circuit and antenna technologies.

However, with such technology comes significant new challenges, an important example of which is the large number of array elements typically required to construct a phased array in such applications. As an illustration, in a vehicle collision avoidance system, obtaining sufficient resolution might require an aperture of roughly $2 \mathrm{~m}$. But in this case a traditional phased array operating at $100 \mathrm{GHz}$ with half-wavelength element spacing would require roughly 1000 antennas, which is daunting to implement.

As a result, there is renewed interest in developing sparse antenna array architectures. Sparse arrays, characterized by average inter-element spacings of greater than one half of the operating wavelength, have been of interest throughout much of the history of phased arrays, garnering a great deal of attention in the early 1960's; see, e.g., [1] and references

Manuscript received November 2012. This work was supported in part by the National Science Foundation under Grant No. CCF-1017772, by the Semiconductor Research Corporation through the Focus Center Research Program's Center for Circuits and System Solutions (C2S2), and by the United States Air Force under Contract No. FA8721-05-C-0002. Opinions, interpretations, conclusions and recommendations are those of the authors and are not necessarily endorsed by the United States Government.

J. Krieger and G. W. Wornell are with the Department of Electrical Engineering and Computer Science, Massachusetts Institute of Technology, Cambridge, MA 02139 (Email: \{jameskrieger,gww\} @ mit.edu).

Y. Kochman is with the School of Computer Science and Engineering, Hebrew University of Jerusalem, Israel (Email: yuvalko@cs.huji.ac.il). therein. The design of general-purpose sparse arrays has typically entailed making basic performance tradeoffs. A wellknown example is the use of "density tapering", which uses a gradually increasing spacing profile as one moves from the center toward the edges of the aperture. These arrays are representative of a class of "thinned" arrays that stretch the aperture associated with a given number of elements to achieve a desired resolution by narrowing the width of the main lobe without introducing additional grating lobes. However, this is obtained at the cost of a significant increase in the side lobe level. In certain applications for which resolution is the key performance metric, these provide a useful design solution. However, in the context of imaging arrays this introduces an unacceptable noise floor.

Another class of sparse arrays, referred to as limited fieldof-view or limited scan arrays, accommodate sparseness by constraining the field-of-view of the array to a commensurately narrow range of angles, since this allows the resulting grating lobes to be effectively ignored. Limiting the field-of-view is accomplished through the use of directive antenna elements, which suppress the grating lobes outside of the angular region of interest. While the associated grating lobes still reduce the array gain, interference is avoided. Because of the narrow field-of-view, however, such arrays must be physically rotated to image a complete scene, which requires considerably mechanical complexity as well as a comparatively static scene. As a result, such designs are not well suited to providing costeffective imaging solutions.

In this paper, we take a different approach, whereby rather than constraining the functionality or performance of the array, we exploit structure in the scene being imaged. In particular, we seek to exploit sparsity in the scene to allow the number of antenna elements to be reduced, i.e., when the scene being imaged is sparse in an appropriate sense-even without knowing where it is sparse-then it is possible to commensurately reduce the number of elements in an imaging array. Moreover, such sparseness is quite common in typical applications. ${ }^{1}$

This approach also has a rich history. Consider, for example, the classical problem of direction-finding with multiple sources, for which the MUSIC algorithm [2], among others, was developed. In this case, it is possible to achieve highresolution with relatively few antenna elements because of the simple structure of scene. Indeed, the number of elements required is typically on the order of the number of sources. Hence, the presence of structure in the environment allows the number of elements to be reduced.

For arrays containing just a few elements, the array design

\footnotetext{
${ }^{1}$ Note that in a typical scene while there are objects at some range in any particular direction, when we use enough bandwidth to sufficiently resolve range as well, we find significant sparseness in the range-azimuth plane.
} 
and image reconstruction can often be fairly straightforward and exploit classical techniques. However, for arrays of even a few dozen elements, such direct approaches quickly become computationally infeasible to design, and impractical to implement. As a result, there is a need to impose useful structure on the array, to enable efficient design and processing.

In our development, we therefore focus on particular structured sparse antenna designs that are comparatively easy to design and for which efficient array processing algorithms can be developed to perform the image reconstruction. Specifically, we focus on "multi-coset" arrays, whose elements are laid out in a quasi-periodic (recurrent nonuniform) pattern, and which can be viewed as a collection of overlapping, staggered sparse uniform arrays.

This architecture, introduced in [3], follows from exploiting the close mathematical relationship between the problem of imaging from a discrete array, and that of reconstructing a bandlimited time-domain waveform from samples. Indeed, our architecture is the counterpart of multi-coset sampling [4]. And while [3] focuses on making the mathematical connection between these domains, the present paper represents a more complete development of the design and analysis of multicoset sparse imaging arrays in their own right.

The paper is organized as follows. Section II defines the scene model and array structure of interest, and introduces some convenient notation. In Section III, the basic multicoset image reconstruction algorithm and its implementation are developed for an ideal array. Section IV incorporates finite-aperture effects and the presence of noise into the analysis, which leads to a novel co-array based procedure for finding robust and efficient multi-coset patterns. Section V develops a methodology for reliably detecting reconstruction failures when the scene density exceeds the level for which an array is designed, preventing false images from being produced. Section VI developes the extension of the methodology to 2-D range-azimuth imaging, which accommodates range-dependent sparsity, and demonstrates the performance on a simulated scene. Finally, Section VII concludes with a discussion of the results and their implications.

\section{Preliminaries}

Throughout this paper, we will focus on horizontally aligned linear arrays of elements located on some subset of collinear lattice points with uniform spacing $d=\lambda / 2$, where $\lambda$ is the operating wavelength of the array. We assume ideal isotropic elements and limit our attention to the horizontal half-plane such that the directional characteristics of the array are completely specified by the angle $\theta$, measured from the broadside direction of the array as shown in Fig. 1.

A standard linear array refers to any array having uniform element spacing of $\lambda / 2$. Substituting $\psi=\sin \theta / 2$, we may express the array response and far-field pattern for a standard array with $N \rightarrow \infty$ elements as a Fourier transform pair,

$$
\begin{aligned}
x[n] & =\int_{0}^{1} X(\psi) e^{j 2 \pi \psi n} d \psi, \quad n \in \mathbb{Z} \\
X(\psi) & =\sum_{n} x[n] e^{-j 2 \pi \psi n}, \quad \psi \in[0,1) .
\end{aligned}
$$

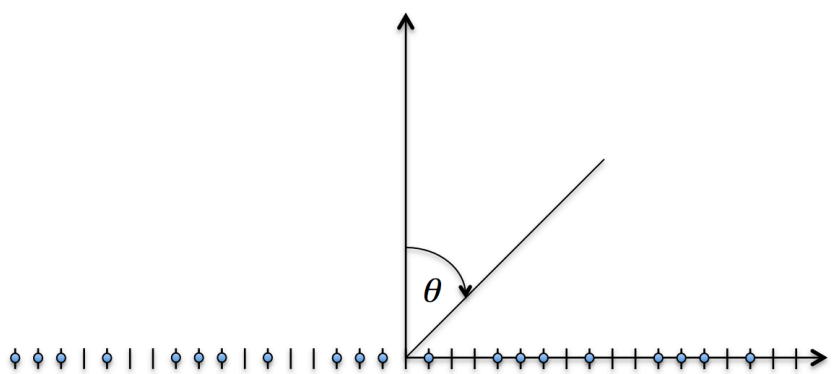

Fig. 1. $(4,7)$ multi-coset array with coset pattern $\mathcal{P}=\{0,1,2,4\}$

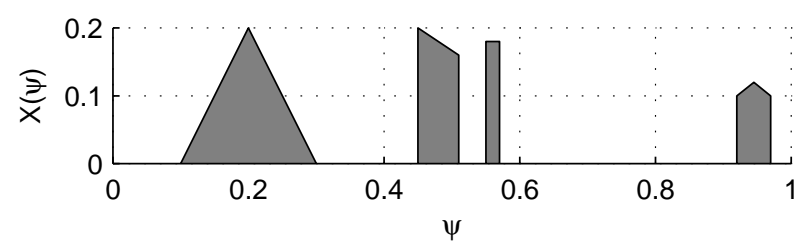

Fig. 2. (5, 10)-sparse scene with $\mathcal{Q}=\{1,2,4,5,9\}$

From the view of array imaging, (1) describes $x[n]$ as the response at element $n$ to a scene consisting of complex valued objects $X(\psi)$. Standard reconstruction of the scene in a given direction $\psi$ is carried out using (2).

We define block sparsity as follows. For any pair of integers $Q \leq L$, we say that a scene is $(Q, L)$-sparse if $X(\psi)=0$ for all $\psi \notin \Psi$, where

$$
\Psi_{\mathcal{Q}}=\bigcup_{k=0}^{Q-1}\left[\frac{q_{k}}{L}, \frac{q_{k}+1}{L}\right)
$$

where the $q_{k}$ are the integer valued elements in the set $\mathcal{Q}=$ $\left\{q_{0}, q_{1}, \ldots, q_{Q-1}\right\}, 0 \leq q_{0}<q_{1}<\cdots<q_{Q-1} \leq L-1$. This set $\mathcal{Q}$ is referred to as the block support of the scene. An example of a $(5,10)$-sparse scene is shown in Fig. 2.

It is possible to exploit scene sparsity to reduce the number of elements for a given aperture required for accurate imaging. In particular, we employ the periodic non-uniform spatial multi-coset sampling scheme, due its particular suitability to block sparse imaging [4]. The multi-coset structured sparse array is composed of a subset of the uniformly spaced standard array described by $x[n]$. For any pair of integers $P \leq L$, a $(P, L)$ multi-coset array is the union of $P$ overlapping subarrays, known as cosets, with inter-element spacing of $L$ times 
greater than the nominal inter-element spacing, such that we refer to $L$ as the coset period. The particular choice of cosets $\mathcal{P}=\left\{p_{0}, p_{1}, \ldots, p_{P-1}\right\}, 0 \leq p_{0}<p_{1}<\cdots<p_{P-1} \leq L-1$, is referred to as the coset pattern of the array. In Fig. 1, an example layout of a $(4,7)$ multi-coset array with coset pattern $\mathcal{P}=\{0,1,2,4\}$ is shown over the lattice points representing the location of elements in a standard linear array.

The coset response for coset $p$ is defined over the complete range of $n$ as being equal to $x[n]$ where a coset element is present, and zero otherwise,

$x^{(p)}[n]=x[n] \sum_{m} \delta[n-(m L+p)], \quad p \in\{0,1, \ldots, L-1\}$.

The corresponding coset image is the Fourier transform of the coset response

$$
X^{(p)}(\psi)=\sum_{n} x^{(p)}[n] e^{j 2 \pi \psi n}, \quad \psi \in[0,1) .
$$

\section{Multi-Coset Imaging: Ideal Setting}

We first review the process for image reconstruction of a block-sparse scene from the response of a sparse multicoset array. In this section, we assume the idealized case of an infinite array aperture and ignore noise. This material will establish the basic notation and serve as a basis in the subsequent analyses for the practical issues covered in later sections.

\section{A. Reconstruction for Known Support}

The individual coset images in (5) will contain $L$ uniformly shifted copies of the original scene due to the aliasing effect cause by the increased element spacing $L \cdot d$. As a result each of the $L$ coset image blocks will contain grating lobes from the remaining $L-1$ blocks. The basic notion of the multi-coset image reconstruction is to extract the entire correct image from the images of multiple cosets in a single block. Combining (1), (2), (4), and (5), the coset image in the first block can be written as a linear combination of the grating lobes of the original scene

$$
X^{(p)}(\psi)=\frac{1}{L} \sum_{q=0}^{L-1} X(\psi+q / L) e^{j 2 \pi p q / L}, \quad \psi \in[0,1 / L)
$$

Defining $Y_{p}(\psi)=X^{(p)}(\psi) H(\psi)$ and $X_{q}(\psi)=X(\psi+$ $q / L) H(\psi)$, where

$$
H(\psi)= \begin{cases}1 & \psi \in[0,1 / L) \\ 0 & \text { otherwise }\end{cases}
$$

the expression in (6) may be generalized to relate all $L$ sectors to all $L$ possible cosets as the linear relation $\mathbf{Y}(\psi)=\mathbf{A X}(\psi)$, where $\mathbf{A}$ is the $L \times L$ inverse discrete Fourier transform matrix with elements $A_{p q}=\frac{1}{L} e^{j 2 \pi p q / L}$. Hence, given the complete set of coset responses $\left\{Y_{p}(\psi)\right\}$, it is straightforward to reconstruct the correct image of the original scene.

Since we are interested in a $(P, L)$ sparse multi-coset array, we must consider instead the length- $P$ vector $\mathbf{Y}_{\mathcal{P}}(\psi)$, composed of the entries of $\mathbf{Y}(\psi)$ indexed by the coset pattern $\mathcal{P}$. Similarly, we define the $P \times L$ matrix $\mathbf{A}_{\mathcal{P}}$ containing the $P$ rows of $\mathbf{A}$ indexed by $\mathcal{P}$. This results in the relation $\mathbf{Y}_{\mathcal{P}}(\psi)=\mathbf{A}_{\mathcal{P}} \mathbf{X}(\psi)$. In this form, we now have an undetermined system, having an infinite number of possible solutions.

For a $(Q, L)$-sparse scene with support $\mathcal{Q}$, the elements of $\mathbf{X}(\psi)$ not indexed by this support will be zero valued, and thus do not contribute to the coset responses. Hence, we may define the $P \times Q$ matrix $\mathbf{A}_{\mathcal{P Q}}$, composed of the columns of $\mathbf{A}_{\mathcal{P}}$ indexed by $\mathcal{Q}$, and the length- $Q$ vector $\mathbf{X}_{\mathcal{Q}}(\psi)$ containing the nonzero entries of $\mathbf{X}(\psi)$. The matrix $\mathbf{A}_{\mathcal{P} \mathcal{Q}}$ is referred to as the measurement matrix. The updated relation becomes

$$
\mathbf{Y}_{\mathcal{P}}(\psi)=\mathbf{A}_{\mathcal{P} \mathcal{Q}} \mathbf{X}_{\mathcal{Q}}(\psi)
$$

If $\mathbf{A}_{\mathcal{P Q}}$ is full rank, the correct image may be reconstructed as

$$
\hat{\mathbf{X}}_{\mathcal{Q}}(\psi)=\mathbf{A}_{\mathcal{P} \mathcal{Q}}^{+} \mathbf{Y}_{\mathcal{P}}(\psi)
$$

where $\mathbf{A}_{\mathcal{P} \mathcal{Q}}^{+}=\left(\mathbf{A}_{\mathcal{P} \mathcal{Q}}^{H} \mathbf{A}_{\mathcal{P} \mathcal{Q}}\right)^{-1} \mathbf{A}_{\mathcal{P} \mathcal{Q}}^{H}$ is the Moore-Penrose pseudo-inverse of the matrix $\mathbf{A}_{\mathcal{P} \mathcal{Q}}$.

The rank of $\mathbf{A}_{\mathcal{P} \mathcal{Q}}$ depends on both $\mathcal{P}$ and $\mathcal{Q}$. A pattern $\mathcal{P}$ which guarantees full rank for any support of length $Q$ is called a universal pattern. As shown in [4], such patterns exist whenever $P \geq Q$. For example, the so-called "bunched" pattern, in which the first $P$ cosets are selected, is generally universal. However, in Section IV, we will see that the universality condition is merely a minimal necessary requirement and does not ensure optimal performance once practical issues such as receiver noise are taken into account.

\section{B. Blind Support Recovery}

In practice, a more robust reconstruction method assumes only a maximum value of $Q$ such that the scene satisfies the block sparsity description in (3) for unknown support $\mathcal{Q}$. This entails the need for a step prior to the image reconstruction in which an estimate of the support $\hat{\mathcal{Q}}$ is determined from the available data. Once the support recovery is complete, the reconstruction can be performed using (9).

The complete blind reconstruction problem can be framed as a compressed sensing (CS) problem which seeks a solution $\hat{\mathbf{X}}(\psi)$ to $\mathbf{Y}_{\mathcal{P}}(\psi)=\mathbf{A}_{\mathcal{P}} \mathbf{X}(\psi)$ having the minimum number of nonzero entries. Since the location of the non-zero entries in $\mathbf{X}(\psi)$ are identically located for all $\psi \in[0,1 / L)$, a good estimator will consider the data over this entire range simultaneously. This support recovery problem may be cast in terms of the setting known in the CS literature as a multiple measurement vectors (MMV) problem, for which support recovery is guaranteed for any $(Q, L)$-sparse scene when $P \geq 2 Q$ [5]. Compared to the known support problem, this implies that blindness necessitates a factor two increase in the minimum number of array elements. However, for a wide class of scenes, this strict requirement may be relaxed.

There exist a variety of specific algorithms suited to the blind support recovery; see, e.g., [6], [7]. For the most part, these operate on the correlation matrix in order to examine the 
entirety of the available coset data,

$$
\begin{aligned}
\mathbf{S}_{\mathbf{Y}_{\mathcal{P}}} & =\int_{0}^{\frac{1}{L}} \mathbf{Y}_{\mathcal{P}}(\psi) \mathbf{Y}_{\mathcal{P}}^{H}(\psi) d \psi \\
& =\mathbf{A}_{\mathcal{P}}\left(\int_{0}^{\frac{1}{L}} \mathbf{X}(\psi) \mathbf{X}^{H}(\psi) d \psi\right) \mathbf{A}_{\mathcal{P}}^{H} \\
& =\mathbf{A}_{\mathcal{P}} \mathbf{S}_{\mathbf{X}} \mathbf{A}_{\mathcal{P}}^{H}
\end{aligned}
$$

it is shown in [8] that support recovery is guaranteed whenever

$$
P \geq 2 Q-\operatorname{rank}\left(\mathbf{S}_{\mathbf{X}}\right)+1 .
$$

Under the assumption that the rows of $\mathbf{X}(\psi)$ form a linearly independent set, $\mathbf{S}_{\mathbf{X}}$ will have rank $Q$, resulting in the support recovery guarantee condition $P \geq Q+1$. In this case, the price of blindness is the addition of a single coset. In general, it is reasonable to expect small levels of correlation between the image contents in the different blocks. As such, $P=Q+1$ may be considered to be the extreme minimum choice, with $P=Q+2$ being, for the most part, a much safer choice for providing reliable estimates of $\hat{\mathcal{Q}}$. The issues of reliable support recovery and SNR dependence will be covered in detail in Section IV.

The support recovery problem may be expressed as a compressed sensing problem as follows. The coset correlation matrix is decomposed into $\mathbf{S}_{\mathbf{Y}_{\mathcal{P}}}=\mathbf{V} \mathbf{V}^{H}$, where $\mathbf{V}$ may be found from the singular value decomposition $\mathbf{S}_{\mathbf{Y}_{\mathcal{P}}}=\mathbf{U} \boldsymbol{\Lambda} \mathbf{U}^{H}$ such that $\mathbf{V}=\mathbf{U} \boldsymbol{\Lambda}^{1 / 2}$. The matrix $\mathbf{V}$ can be expressed as $\mathbf{V}=\mathbf{A}_{\mathcal{P}} \mathbf{W}$. Since $\mathbf{A}_{\mathcal{P}}$ is a $P \times L$ matrix $(P \leq L)$, there is no unique solution to this relation. The compressed sensing problem seeks the solution $\mathbf{W}_{0}$ that minimizes the number of rows having non-zero entries. This particular $\mathcal{L}_{0}$-minimization problem may be replaced by a computationally preferable $\mathcal{L}_{1}$ minimization problem. For this we define the length- $L$ vector w with entries equal to the $\ell_{2}$-norm of the corresponding rows of W. With this, the problem becomes

$$
\text { minimize }\|\mathbf{w}\|_{1} \quad \text { s.t. } \mathbf{V}-\mathbf{A}_{\mathcal{P}} \mathbf{W}=\mathbf{0} .
$$

Solutions to the $\mathcal{L}_{1}$-minimization problem may still require more computation time than desired for a dynamic imaging system. An alternative solution originally proposed in [4] is based on the MUSIC direction finding algorithm [9]. The basic algorithm is as follows. In the absence of noise, the correlation matrix will have $Q$ out of $P$ non-zero eigenvalues. The eigenvector matrix is partitioned as $\mathbf{U}=\left[\mathbf{U}_{S} \mathbf{U}_{N}\right]$, where the $L \times(P-Q)$ matrix $\mathbf{U}_{N}$ contains the eigenvectors corresponding to the zero valued eigenvalues. This matrix represents the noise subspace of $\mathbf{U}$. Each of the $L$ columns of $\mathbf{A}_{\mathcal{P}}$ is projected onto the noise subspace. The columns corresponding to the active sectors contained within the support $\mathcal{Q}$ will lie in the orthogonal subspace spanned by $\mathbf{U}_{S}$ and hence will have zero projection onto $\mathbf{U}_{N}$. The recovered support $\hat{\mathcal{Q}}$ will contain the indices of these columns. Thus, defining the columns of $\mathbf{A}_{\mathcal{P}}$ and $\mathbf{U}$ as $\mathbf{a}_{q}$ and $\mathbf{u}_{m}$, the algorithm evaluates the null spectrum

$$
D_{\text {MUSIC }}(q)=\sum_{m=Q+1}^{P}\left|\mathbf{a}_{q}^{H} \mathbf{u}_{m}\right|^{2}
$$

and selects $\hat{\mathcal{Q}}$ as the values of $q$ corresponding to the $Q$ smallest values of $D_{\mathrm{MUSIC}}(q)$.

\section{System Implementation}

It is straightforward to implement this system in the array domain, allowing for operation on the coset responses $x^{(p)}[n]$ directly. The inverse Fourier transform of (8) yields the array domain form

$$
\mathbf{y}_{\mathcal{P}}[n]=\mathbf{A}_{\mathcal{P} \mathcal{Q}} \mathbf{x}_{\mathcal{Q}}[n]
$$

and has the similar reconstruction $\hat{\mathbf{x}}_{\mathcal{Q}}[n]=\mathbf{A}_{\mathcal{P} \mathcal{Q}}^{+} \mathbf{y}_{\mathcal{P}}[n]$. The reconstruction is shown in Fig. 3. The coset responses are first passed through the filter $h[n]$ associated with $H(\psi)$ to form the output vector $\mathbf{y}_{\mathcal{P}}[n]$. From the array domain perspective, the entries of $\mathbf{y}_{\mathcal{P}}[n]$ are the interpolated coset responses, having the $L-1$ values between each coset element provided as a result of the interpolating filter $h[n]$. To the vector of interpolated coset responses, we apply the $Q \times P$ matrix $\mathbf{A}_{\mathcal{P} \mathcal{Q}}^{+}$ to obtain the $\hat{\mathbf{x}}_{\mathcal{Q}}[n]$. Note that, in the image domain, the $X_{q}(\psi)$ are related to the original scene by

$$
X(\psi)=\sum_{q=0}^{L-1} X_{q}(\psi-q / L) .
$$

Hence, the output of the matrix multiplication operation is the reconstructed response for shifted copies of the blocks contained in the support $\mathcal{Q}$ and the complete array response $\hat{x}[n]$ may be formed in the array domain by modulating and summing the $Q$ contributions from $\hat{\mathbf{x}}_{\mathcal{Q}}[n]$ as shown in Fig. 3 .

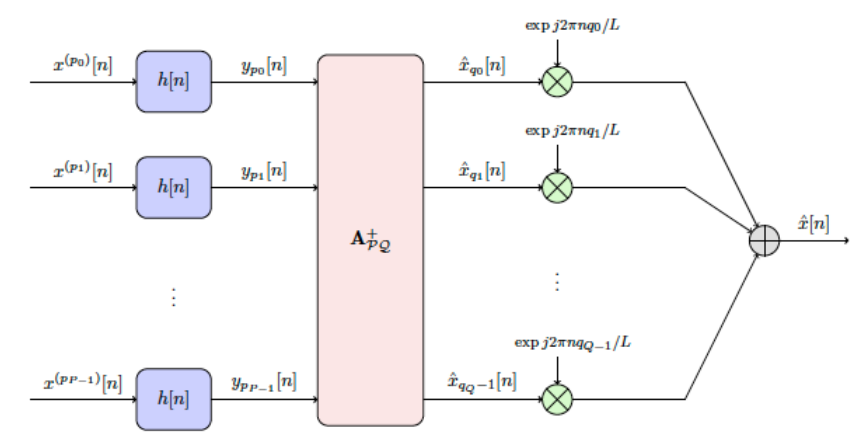

Fig. 3. Multi-coset reconstruction processing chain.

The estimated support $\hat{\mathcal{Q}}$ may be found by tapping the $\mathbf{y}_{\mathcal{P}}[n]$ prior to the reconstruction operation. The correlation matrix can also be computed directly from the coset responses in the array domain from

$$
\left\{\mathbf{S}_{\mathbf{Y}_{\mathcal{P}}}\right\}_{l k}=\left\langle x_{p_{l}}[n], x_{p_{k}}[n]\right\rangle=\sum_{n} x_{p_{l}}[n] x_{p_{k}}^{*}[n] .
$$

This estimate is then returned to be used in the matrix multiplication and the subsequent delays. 


\section{PRACTICAL IMPLEMENTATION ISSUES}

In Section III, our development ignored the important issues of finite aperture length and the presence of noise. Because of the reality of both these issues in any practical array system, the block sparsity described in (3) can never be achieved in a strict sense. In this section, we will discuss these issues and their consequences.

\section{A. Finite Aperture Effects}

In Section III, the effects of finite aperture lengths were ignored. A finite aperture causes the main lobe beamwidth to increase and also produces sidelobes. The lower resolution resulting from increased beamwidth turns point targets into unresolved responses spread over a region of size $\Delta \psi \approx 2 / L$. The sidelobes, while at much lower amplitude, cause the response to spread even wider. These effects are well understood in standard array processing, however, they present additional difficulties to the multi-coset array since targets in the supported blocks $\mathcal{Q}$ will effectively have a "spillover" into the neighboring blocks, violating the original sparsity assumption in (3).

For a fixed aperture length, there is a direct tradeoff between the coset period $L$ and the number of coset periods $M$ with $N=M L$ taking values less than or equal to the total number of elements in a standard array covering the extent of the available aperture. Since the block-scene density $\rho_{s, L}=Q / L$ will converge to the actual scene density $\rho_{s}$ from above as $L \rightarrow \infty$, this motivates a choice of large $L$. However, the performance will decrease as the number of coset periods decreases. This is due mainly to two reasons. First, the number of coset periods is similar to the number of "snapshots" for estimating the support. Second, the leakage associated with finite array lengths will be increasingly troublesome as the scene blocks become more narrow and the spillover energy fills increasingly larger portions of otherwise empty blocks.

As will be discussed shortly, the reconstruction and recovery algorithms have a certain degree of robustness to the situation in which (3) is violated by the presence of non-trivial levels of noise outside of the supported region $\Psi_{\mathcal{Q}}$. From the perspective of the image processing algorithm, the leakage out of $\Psi_{\mathcal{Q}}$ is similar to noise. Hence, this effect is relatively benign as long as the spillover is not noticeably more significant than the noise level. As the primary motivation for this work is to determine design solutions for large $N$, we expect a certain degree of flexibility in the choice of $L$ and $M$ such that this will typically be the case.

In general, standard low-sidelobe tapers used in array processing will mitigate the spillover effect and help to make possible the use of aggressively sparse array designs at high SNRs. For the cases when spillover can simply not be ignored, a modified version of the array-domain reconstruction process is described in [3]. It is shown, that the finite-aperture effects may be reduced to any desired level by dedicating a certain portion of the array to the task (thus reducing resolution).

\section{B. Noise Effects}

The presence of noise affects the development of Section III in a few ways. The support condition in (3) no longer holds and there is no exact sparse solution to $\mathbf{Y}_{\mathcal{P}}(\psi)=\mathbf{A}_{\mathcal{P} \mathcal{Q}} \mathbf{X}_{\mathcal{Q}}(\psi)$, requiring modifications to the recovery algorithms. Below some threshold SNR (TSNR), the recovery algorithms will be unable to reliably estimate the correct support $\mathcal{Q}$. This will of course depend on the specifics of the scene density, and as we will see, the coset pattern $\mathcal{P}$. Also important is how the reconstruction SNR (RSNR) using the sparse multi-coset array compares with the SNR of an image obtained using a standard array.

1) Reconstruction noise amplification: We begin by showing how the RSNR is related to the condition number of the particular measurement matrix $\kappa\left(\mathbf{A}_{\mathcal{P} \mathcal{Q}}\right)$. We first examine the effect of the coset pattern $\mathcal{P}$ on the noise in the reconstructed image. Assume for now that we are in the high SNR regime for which the true support $\mathcal{Q}$ can be reliably estimated. To facilitate tractable analysis, the array response is modeled as a superposition of the original response $x[n]$ corresponding to stationary gaussian signal $X(\psi)$ satisfying (3) for the set $\mathcal{Q}$ and a gaussian noise response $z[n]$ associated with $Z(\psi)$ evenly distributed over $\psi \in[0,1)$. The signal and noise energies are given by $S_{0}=E\left[\int_{0}^{1}|X(\psi)|^{2} d \psi\right]$ and $N_{0}=E\left[\int_{0}^{1}|Z(\psi)|^{2} d \psi\right]$, respectively. Defining $\mathbf{Z}(\psi) \in \mathbb{C}^{L}$, where $\{\mathbf{Z}(\psi)\}_{q}=Z(\psi+q / L)$, the coset response in the image domain is

$$
\begin{aligned}
\mathbf{Y}_{\mathcal{P}}(\psi) & =\mathbf{A}_{\mathcal{P}}(\mathbf{X}(\psi)+\mathbf{Z}(\psi)) \\
& =\mathbf{A}_{\mathcal{P} \mathcal{Q}} \mathbf{X}_{\mathcal{Q}}(\psi)+\mathbf{A}_{\mathcal{P}} \mathbf{Z}(\psi)
\end{aligned}
$$

Proceeding as in the noiseless case, the resultant noisy reconstruction is given by

$$
\begin{aligned}
\hat{\mathbf{X}}_{\mathcal{Q}}(\psi) & =\mathbf{A}_{\mathcal{P} \mathcal{Q}}^{+} \mathbf{Y}_{\mathcal{P}}(\psi) \\
& =\mathbf{X}_{\mathcal{Q}}(\psi)+\mathbf{A}_{\mathcal{P} \mathcal{Q}}^{+} \mathbf{A}_{\mathcal{P}} \mathbf{Z}(\psi)
\end{aligned}
$$

This reconstructed image contains the original image plus a modified noise component. As such, the reconstructed signal energy $S_{r}=S_{0}$, and the reconstructed noise energy is

$$
\begin{aligned}
N_{r} & =E\left[\int_{0}^{1}\left\|\mathbf{A}_{\mathcal{P} \mathcal{Q}}^{+} \mathbf{A}_{\mathcal{P}} \mathbf{Z}(\psi)\right\|^{2} d \psi\right] \\
& =\operatorname{Tr}\left(\left(\mathbf{A}_{\mathcal{P} \mathcal{Q}^{+}}^{+} \mathbf{A}_{\mathcal{P}}\right) E\left[\mathbf{S}_{Z}\right]\left(\mathbf{A}_{\mathcal{P}{ }_{\mathcal{Q}}^{+}} \mathbf{A}_{\mathcal{P}}\right)^{H}\right) .
\end{aligned}
$$

The original noise energy $N_{0}$ is spread evenly over the $L$ diagonal elements of $E\left[\mathbf{S}_{Z}\right]$, so the above expression becomes

$$
N_{r}=\operatorname{Tr}\left(\mathbf{A}_{\mathcal{P} \mathcal{Q}}^{+} \mathbf{A}_{\mathcal{P}} \mathbf{A}_{\mathcal{P}}^{H} \mathbf{A}_{\mathcal{P} \mathcal{Q}}^{+, H}\right) N_{0} / L
$$

The rows of $\mathbf{A}_{\mathcal{P}}$ are taken from the $L \times L$ IDFT matrix, hence $\mathbf{A}_{\mathcal{P}} \mathbf{A}_{\mathcal{P}}^{H}=\mathbf{I}_{P} / L$, and

$$
N_{r}=\operatorname{Tr}\left(\mathbf{A}_{\mathcal{P} \mathcal{Q}}^{+} \mathbf{A}_{\mathcal{P} \mathcal{Q}}^{+, H}\right) N_{0} / L^{2}
$$

Recalling that $S_{r}=S_{0}$, the reconstruction SNR (RSNR) is the original SNR reduced by a factor given by the noise amplification

$$
\frac{N_{r}}{N_{0}}=\frac{\left\|\mathbf{A}_{\mathcal{P} \mathcal{Q}}^{+}\right\|_{F}^{2}}{L^{2}} .
$$


where $\|\cdot\|_{F}$ is the Frobenius matrix norm,

$$
\begin{aligned}
\left\|\mathbf{A}_{\mathcal{P} \mathcal{Q}}^{+}\right\|_{F}^{2} & =\sum_{j=0}^{P-1} \sum_{i=0}^{Q-1}\left|\left[\mathbf{A}_{\mathcal{P} \mathcal{Q}}^{+}\right]_{i j}\right|^{2} \\
& =\sum_{i=0}^{Q-1}\left|\sigma_{i}\left(\mathbf{A}_{\mathcal{P} \mathcal{Q}}^{+}\right)\right|^{2}
\end{aligned}
$$

and the $\sigma_{i}\left(\mathbf{A}_{\mathcal{P} \mathcal{Q}}^{+}\right)$are the $Q$ non-zero singular values of $\mathbf{A}_{\mathcal{P} \mathcal{Q}}^{+}$. From the properties of the pseudo-inverse, these singular values are the reciprocals of the singular values of $\mathbf{A}_{\mathcal{P} \mathcal{Q}}$. Further, since $\mathbf{A}_{\mathcal{P} \mathcal{Q}}$ is a $P \times Q$ matrix with each entry having magnitude $1 / L$, it follows that

$$
\left\|\mathbf{A}_{\mathcal{P} \mathcal{Q}}\right\|_{F}^{2}=\frac{P Q}{L^{2}}=\sum_{i=0}^{Q-1}\left|\sigma_{i}\left(\mathbf{A}_{\mathcal{P} \mathcal{Q}}\right)\right|^{2} .
$$

While the sum in (25) is fixed for a given $L, P$, and $Q$, the sum in (24) may very greatly, depending on the distribution of the singular values of the measurement matrix. Specifically, if the least of $\sigma_{i}\left(\mathbf{A}_{\mathcal{P Q}}\right)$ are particularly small, the noise amplification can become extremely large. As a result, the expected RSNR, for the most part, can be understood in terms of the ratio of the maximum to the minimum singular values, or condition number of the measurement matrix $\kappa\left(\mathbf{A}_{\mathcal{P} \mathcal{Q}}\right)$. Hence, in the selection of the coset pattern, it is desirable to select $\mathcal{P}$ such that $\kappa\left(\mathbf{A}_{\mathcal{P} \mathcal{Q}}\right)$ takes relatively small values for all support sets $\mathcal{Q}$.

2) Support recovery reliability: Regarding the support recovery portion of the reconstruction, it is necessary to consider how the choice of coset pattern affects the recovery reliability. Before discussing this, we first will examine how the recovery algorithms may be modified to handle the presence of noise. For the optimization problem, the constraint must be relaxed in order to obtain a sparse solution. With the MUSIC algorithm approach, difficulties arise due to the finite array lengths that must be employed in practice. The matrices $\mathbf{U}_{S}$ and $\mathbf{U}_{N}$ no longer accurately partition the signal and noise subspaces and the eigenvalues associated with $\mathbf{U}_{N}$ take on non-zero values. The situation is complicated further when the number of occupied blocks $Q$ is unknown. In these cases, an intermediate step must be included to estimate the signal subspace dimension $\hat{Q}$. This may be accomplished through direct thresholding of the eigenvalues. More sophisticated approaches are described in [10]. When $\hat{Q}>Q$, two issues arise. The estimated dimensionality of the noise subspace $P-\hat{Q}$ is reduced making the recovery estimate less accurate. Also, the matrix $\mathbf{A}_{\mathcal{P} \hat{\mathcal{Q}}}$ used in the reconstruction will be more poorly conditioned, resulting in increased noise amplification.

We have found a weighted version of the MUSIC algorithm based on the eigenvalue method [11]. The eigenvalue method accounts for errors due to finite sample sets by weighing the projections onto each subspace direction more heavily for smaller eigenvalues

$$
D_{e v}(q)=\sum_{m=Q+1}^{P} \frac{1}{\lambda_{m}}\left|\mathbf{a}_{q}^{H} \mathbf{u}_{m}\right|^{2} .
$$

Again, this requires some estimate of $Q$. In our modification, we avoid the need for this estimate at this point by weighing the entire column space of $\mathbf{U}$

$$
\tilde{D}_{e v}(q)=\sum_{m=1}^{P}\left(\frac{1}{\lambda_{m}^{1 / 2}}-\frac{1}{\lambda_{1}^{1 / 2}}\right)^{2}\left|\mathbf{a}_{q}^{H} \mathbf{u}_{m}\right|^{2} .
$$

Numerical simulations were performed to compare the different recovery algorithms. A representative example of the results is shown in Fig. 4. In these simulations, a coset period of $L=19$ with $P=9$ active cosets was selected. The coset pattern is fixed at $\mathcal{P}=\{0,1,2,3,5,7,12,13,16\}$, selected by the design algorithm described in the following section. To gauge the recovery performance of the basic MUSIC, eigenvalue-MUSIC, modified-eigenvalue-MUSIC, and $\mathcal{L}_{1}$-minimization algorithms, each was applied to 1000 randomly generated scenes in which $Q=7$ active sectors supported by $\mathcal{Q}$ were selected at random. Gaussian noise was added to each scene, evenly distributed over the entire range of $\psi$. This was repeated over a range of SNRs. A successful recovery was declared when the $Q$ most likely active sectors as estimated by the respective algorithm matched exactly to the $Q$ sectors contained in $\mathcal{Q}$. While the $\mathcal{L}_{1}$ algorithm gave the best result, our modified MUSIC algorithm performed nearly as well, taking longer to transition, but reaching full reliability at a similar TSNR, while requiring considerably less computation time. Most notable is the improvement beyond the established MUSIC algorithms.

We now need to consider how the choice of $\mathcal{P}$ effects the recovery probability. Because the received noise is independent of the support $\mathcal{Q}$, it does not follow directly that a well conditioned measurement matrix $\mathbf{A}_{\mathcal{P} \mathcal{Q}}$ plays a significant role in this. We propose an alternate approach, inspired by the Minimum Redundancy Linear Arrays (MRLA) introduced in [12]. These arrays are designed so that the number of sensor pairs having identical spacings is minimized in an effort to yield the best representation of the full correlation matrix with the least number of elements. Our approach is similar, with the notable distinction of an accounting for the periodic nature of the multi-coset array. This results in the distance between pairs of cosets to be specified by the minimum distance within either the same, or the neighboring coset periods. Specifically,

$$
\gamma_{l k}=\min \{|l-k|, L-|l-k|\} .
$$

To understand the reasoning for this design approach, consider the correlation matrix associated with all $L$ cosets

$$
\mathbf{S}_{\mathbf{Y}}=\mathbf{A} \mathbf{S}_{\mathbf{X}} \mathbf{A}^{H} \text {. }
$$

For the moment, consider the model in which the $Q$ functions $\left\{X_{q}(\psi)\right\}$ comprise a linearly independent set, such that

$$
\left\{\mathbf{S}_{\mathbf{X}}\right\}_{m n}=\int_{0}^{\frac{1}{L}} X_{m}(\psi) X_{n}^{*}(\psi) d \psi=\sigma_{m}^{2} \delta_{m n},
$$

where $\sigma_{m}^{2}=\int_{0}^{1 / L}\left|X_{m}(\psi)\right|^{2} d \psi$ is the signal energy from block $m$. In this case, the full correlation matrix will have a Hermitian-circulant structure

$$
\left\{\mathbf{S}_{\mathbf{Y}}\right\}_{l k}=\frac{1}{L^{2}} \sum_{q=0}^{L-1} \sigma_{q}^{2} e^{j 2 \pi(l-k) q / L} .
$$




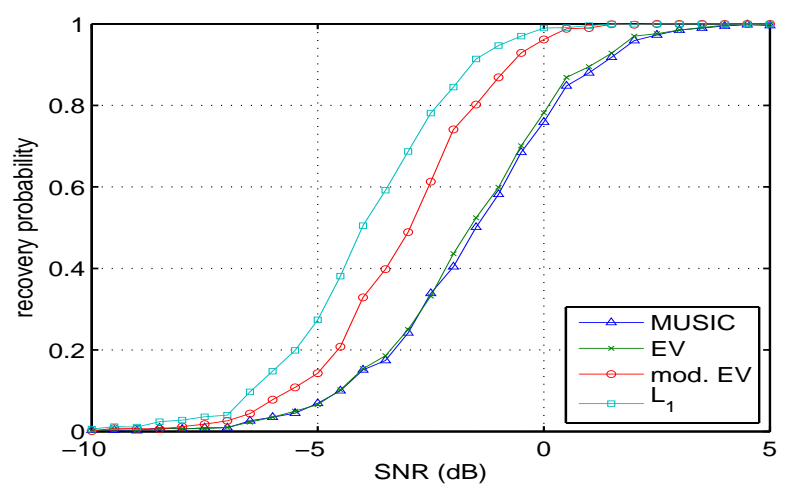

(a)

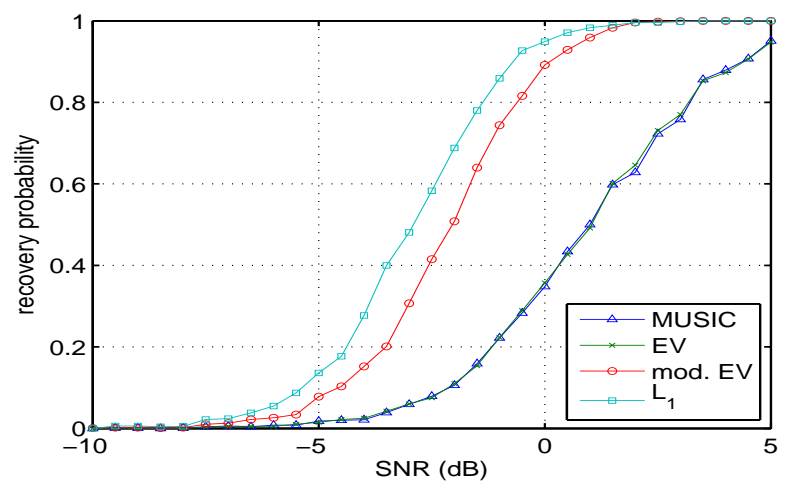

(b)

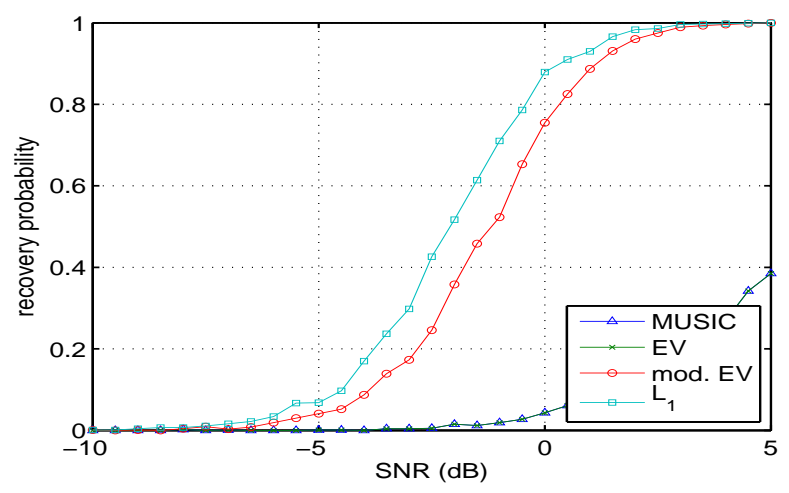

(c)

Fig. 4. Empirical recovery probability versus SNR for $L=19, P=9$, and (a) $Q=6$ (b) $Q=7$ (c) $Q=8$

From (31), the dependence of the matrix entries on the relative spacing between elements indicates the importance of the pairwise spacings as represented by the $2(L-1)$ off-diagonals. Specifically, the information contained in $\mathbf{S}_{\mathbf{Y}}$ can be obtained by representing each of the possible spacings a single time. The symmetries in the Hermitian-circulant structure reduce the number of unknowns by another factor of four, suggesting the entire matrix could be represented by only $\lceil(L-1) / 2\rceil$ unknowns. In reality, there will be measurement noise and some correlation between the different sectors, and as such $\mathbf{S}_{\mathbf{Y}}$ will vary to some extent along each diagonal. As such, multiple occurrences of a particular pairwise spacing can be interpreted as multiple samples of noisy data. Hence, this suggests a design with evenly distributed spacings.
To determine the number of times each pairwise spacing is found in a particular pattern $\mathcal{P}$, we define the length- $L$ binary selection vector $\mathbf{s}_{\mathcal{P}}$ and compute

$$
c(\gamma)=\sum_{\gamma_{l k}=\gamma} s_{l} s_{k}, \quad 1 \leq \gamma \leq(L-1) / 2 .
$$

This function is a modified version of the co-array referred to in the original literature. Since the total number of spacings is identical for all patterns of length $P$, the coset pattern having the co-array with the smallest $\ell_{2}$-norm will be selected.

\section{Design procedure and coset pattern selection}

We will now look at the multi-coset array design process for a linear aperture of length $N$, measured in half-wavelengths, for the situation in which an assumption is made regarding the scene density $\rho_{s}$. The first step is to select the coset period $L$. For simplicity, we will assume that $N$ is near enough to an integer multiple of $L$. The block-scene density $\rho_{s, L}=Q / L$ converges to $\rho_{s}$ from above as $L \rightarrow \infty$. However, the performance will decrease as the number of coset periods decreases as described in Sec. IV-A. As such, in general it is best to choose the smallest $L$ for which the block density has mostly converged.

The array density is then determined by selecting the number of active cosets $P$. The extreme minimum for recovery to remain possible is $P_{\min }=Q+1 \geq \rho_{s} L+1$. As discussed in Section III-B, it is advantageous to select $P>P_{\min }$. This not only makes the system less susceptible to errors, but additionally the support recoverability at low SNR improves most dramatically with the addition of an extra coset from $P=Q+1$ to $P=Q+2$.

Once $L$ and $P$ have been selected, the coset pattern must be determined. From Section IV-B, we have the possible design criteria of either minimizing the condition number over the entire set of supports or minimizing the $\ell_{2}$-norm of the coarray associated with $\mathcal{P}$. We first will outline the optimization of $\mathcal{P}$ based on these two design goals and will then compare the results.

The pattern $\mathcal{P}_{C N}^{*}$ is optimized for $\kappa\left(\mathbf{A}_{\mathcal{P} \mathcal{Q}}\right)$ as follows.

1) Generate the set $\mathcal{S}_{\mathcal{P}}$ containing $N_{\mathcal{P}}$ potential coset patterns

- As a baseline, this can contain up to $L$-choose- $P$ patterns

- This may be reduced significantly by removing patterns which are repeated due to symmetries (reflection, circular shifts).

2) Similarly, generate the set $\mathcal{S}_{\mathcal{Q}}$ containing $N_{\mathcal{Q}}$ block supports

3) For each $\mathcal{P} \in \mathcal{S}_{\mathcal{P}}$,

- calculate $\kappa\left(\mathbf{A}_{\mathcal{P Q}}\right)$ for each of the $N_{\mathcal{Q}}$ supports

- store $\kappa_{\mathcal{P}}^{\max }=\max _{\mathcal{Q} \in \mathcal{S}_{\mathcal{Q}}}\left(\kappa\left(\mathbf{A}_{\mathcal{P} \mathcal{Q}}\right)\right)$

4) Select $\mathcal{P}_{C N}^{*}=\operatorname{argmin}_{\mathcal{P} \in \mathcal{S}_{\mathcal{P}}}\left(\kappa_{\mathcal{P}}^{\max }\right)$ as the coset pattern with the minimum output from step (3)

The pattern $\mathcal{P}_{C O}^{*}$ is optimized for the co-array design as follows.

1) Generate the set $\mathcal{S}_{\mathcal{P}}$ containing $N_{\mathcal{P}}$ potential coset patterns 
2) For each $\mathcal{P}$

- create the length- $L$ vector $\mathbf{s}_{\mathcal{P}}$ consisting of entries equal to 1 at the locations contained in $\mathcal{P}$ and 0 otherwise.

- calculate $\mathbf{c}_{\text {temp }}=\operatorname{conv}\left(\left[\mathbf{s}_{\mathcal{P}} \mathbf{s}_{\mathcal{P}}\right], \overline{\mathbf{s}}_{\mathcal{P}}\right)$, where $\overline{\mathbf{s}}_{\mathcal{P}}$ is a flipped copy of $\mathbf{s}_{\mathcal{P}}$.

- select the relevant portion of the convolution output $\mathbf{c}=\mathbf{c}_{\text {temp }}(L+1: L+(L-1) / 2)$

- store $\|\mathbf{c}\|_{2}$

3) Select $\mathcal{P}_{C O}^{*}$ as the coset pattern with the minimum output from step (2)

4) Check $\kappa_{\mathcal{P}}^{\max }$ for $\mathcal{P}_{C O}^{*}$ against the set $\mathcal{S}_{\mathcal{Q}}$ of possible supports $\mathcal{Q}$

5) If necessary, select the next minimum $\mathcal{P}$ and repeat until a satisfactory conditioning is found

To begin, we examine a set of $(L, P)$ pairs for which the number of unique element pairs $P(P-1) / 2$ is an integer multiple of the number of possible spacings $(L-1) / 2$. This condition allows the special quality of having a perfectly flat co-array distribution. Examples of coset patterns fitting this description are shown in Table I, along with the array density $\rho_{A}$, and the co-array c. To clarify the concepts introduced so far, consider the $(4,7)$-sparse array in the second entry. While the coset pattern may repeat for any number of periods depending on the array length, the array density is fixed, having approximately $57 \%$ of the number of elements contained in a standard array of the same length. Each entry of the coarray $\mathbf{c}=[222]$ is a count of the number of times a particular pairwise spacing occurs for $\gamma=1(0 \leftrightarrow 1,1 \leftrightarrow 2), \gamma=2$ $(0 \leftrightarrow 2,2 \leftrightarrow 4)$, and $\gamma=3(1 \leftrightarrow 4,4 \leftrightarrow 0)$, where the spacing between cosets 4 and 0 "wraps around" and can be thought of as being measured between successive coset periods.

TABLE I EXAMPLES OF COSET PATTERNS $\mathcal{P}_{C O}^{*}$.

\begin{tabular}{c|c|c|c|c}
$L$ & $P$ & $\rho_{A}$ & $\mathcal{P}_{C O}^{*}$ & $\mathbf{c}$ \\
\hline \hline 7 & 3 & 0.43 & $\{013\}$ & {$[111]$} \\
7 & 4 & 0.57 & $\{0124\}$ & {$[222]$} \\
11 & 5 & 0.45 & $\{01247\}$ & {$[2222]$} \\
11 & 6 & 0.55 & $\{012457\}$ & {$[33333]$} \\
13 & 4 & 0.31 & $\{0139\}$ & {$[111111]$} \\
13 & 9 & 0.69 & $\{0123457910\}$ & {$[666666]$} \\
19 & 9 & 0.47 & $\{012357121316\}$ & {$[444444444]$} \\
19 & 10 & 0.53 & $\{01235712131516\}$ & {$[555555555]$}
\end{tabular}

For these values of $L$ and $P$, it is computationally feasible to determine the condition number of the measurement matrix $\kappa\left(\mathbf{A}_{\mathcal{P} \mathcal{Q}}\right)$ over the sets $\mathcal{S}_{\mathcal{P}}$ and $\mathcal{S}_{\mathcal{Q}}$. From these results, the maximum condition number $\kappa_{\mathcal{P}}^{\max }$ over all $\mathcal{Q}$ of length $Q=$ $P-1$ is determined for each $\mathcal{P}$. The results for $\kappa_{\mathcal{P}_{C N}^{*}}^{\max }, \kappa_{\mathcal{P}_{C}^{*}}^{\max }$, and $\kappa_{\mathcal{P}_{B U}}^{\max }$ are shown in Table II for each of the $(L, P)$ pairs in Table I. Here $\mathcal{P}_{B U}=\{0,1, \ldots, P-1\}$ refers to the bunched pattern mentioned in Section III-A. This pattern is included for the sake of reference to demonstrate that while universality with respect to rank may be guaranteed for certain patterns, this does not guarantee anything other than that the condition number of the measurement matrix will be finite for all $\mathcal{Q}$.

The entries in Table II for which $\kappa_{\mathcal{P}_{C N}^{*}}^{\max }$ and $\kappa_{\mathcal{P}_{C O}^{*}}^{\max }$ match show the cases for which the co-array optimization results in
TABLE II

MAXIMUM CONDITION NUMBERS, $Q=P-1$.

\begin{tabular}{c|c|c|c|c}
$L$ & $P$ & $\kappa_{\mathcal{P}_{C N}^{*}}^{\max }$ & $\kappa_{\mathcal{P}_{C O}^{*}}^{\max }$ & $\kappa_{\mathcal{P}_{B U}}^{\max }$ \\
\hline \hline 7 & 3 & 1.31 & 1.66 & 2.64 \\
7 & 4 & 2.18 & 2.18 & 3.60 \\
11 & 5 & 4.24 & 4.24 & 17.54 \\
11 & 6 & 5.17 & 5.17 & 20.22 \\
13 & 4 & 2.75 & 3.26 & 15.85 \\
13 & 9 & 6.49 & 6.49 & 33.25 \\
19 & 9 & 13.54 & 13.54 & 1063.63 \\
19 & 10 & 13.93 & 13.93 & 1154.08
\end{tabular}

the same pattern found through the more exhaustive condition number search. For these cases, the poor conditioning of the bunched patterns is quite clear. However, the large, yet finite, maximum condition number for the bunched patterns agrees with the earlier stipulation that these represent universal patterns. These results demonstrate that the co-array coset pattern tends to be well, if not optimally conditioned.

To compare the effect of the type of pattern on the low-SNR recovery probability, numerical simulations of the same type used to compare algorithm performance in Fig. 4 were performed for a many different combinations of $L, P$, and $Q$ for the different coset pattern types. Selecting the cases in Table II for which the condition number and co-array optimizations did not yield identical coset patterns, the recovery probability as a function of SNR for the $(3,7)$ and $(4,13)$ sparse arrays are shown in Fig.5. In these, and any other choice of $(P, L)$ for which the simulation was conducted, the co-array pattern yielded the lowest TSNR for reliable support recovery.

\section{Support Recovery Failure Detection}

In a dynamic scene environment, it is reasonable to expect changes in the scene density as well as the SNR. As we have shown, both of these quantities effect the support recovery reliability of the multi-coset imaging array system. The value of utilizing the maximum amount of aperture with a minimal number of array elements motivates a desire to operate near the threshold points at which either of these two issues may arise. Hence, it is of great importance to have some indication as to whether the reconstructed image should be trusted, particularly when integrated into a larger system in which decision making processes occur. While some auxiliary analysis may be employed to ensure changes in the image output fit some reality-based model, the benefit of having a self-contained error indication feature included within the processing algorithm is clear. In this section, we develop such a technique based on the concept of back-projection error (BPE).

Consider the $(P, L)$ multi-coset array with coset pattern $\mathcal{P}$ and a $(Q, L)$-sparse scene with support $\mathcal{Q}$, where both $Q$ and $\mathcal{Q}$ are unknown. In the support recovery stage, the received information contained in $\mathbf{Y}_{\mathcal{P}}(\psi)$ is used to obtain an estimate of the support $\hat{\mathcal{Q}}$. Using the estimated support, the image is reconstructed as $\hat{\mathbf{X}}_{\hat{\mathcal{Q}}}(\psi)=\mathbf{A}_{\mathcal{P} \hat{\mathcal{Q}}}^{+} \mathbf{Y}_{\mathcal{P}}(\psi)$. Since the true $\mathbf{X}_{\mathcal{Q}}(\psi)$ is unknown, we use a back-projection onto the space 


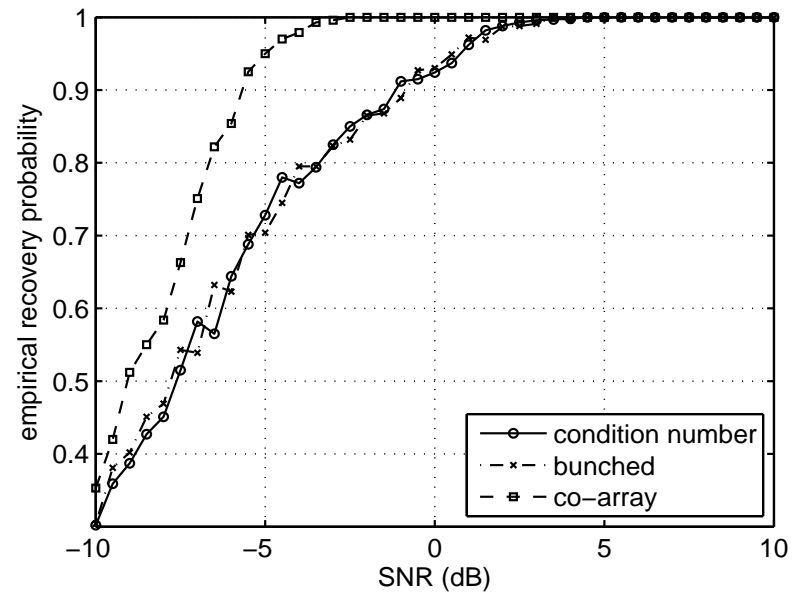

(a)

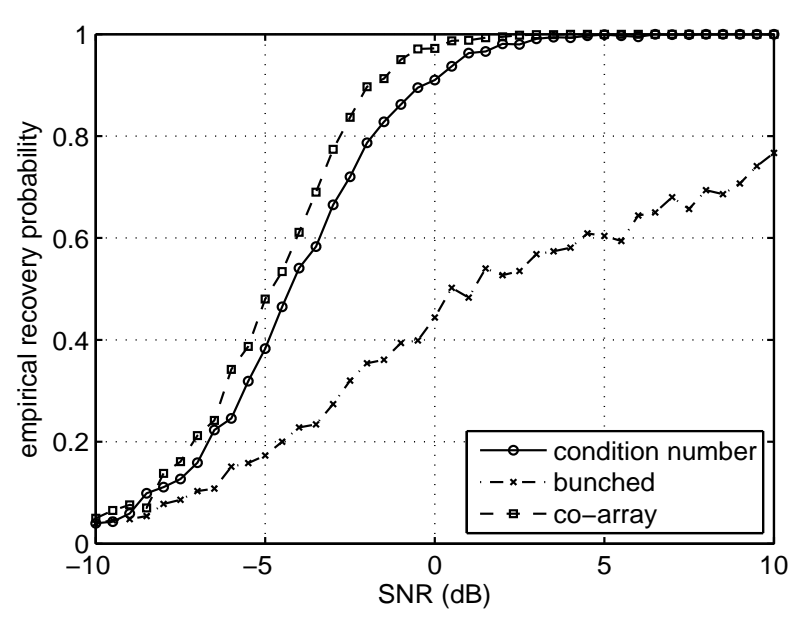

(b)

Fig. 5. Recovery probability vs SNR for (a) $(L, P, Q)=(7,3,2)$ and (b) $(L, P, Q)=(13,4,3)$

spanned by $\hat{\mathcal{Q}}$ for comparison to the original coset response

$$
\begin{aligned}
\hat{\mathbf{Y}}_{\mathcal{P} \hat{\mathcal{Q}}}(\psi) & =\mathbf{A}_{\mathcal{P} \hat{\mathcal{Q}}} \hat{\mathbf{X}}_{\hat{\mathcal{Q}}}(\psi) \\
& =\mathbf{A}_{\mathcal{P} \hat{\mathcal{Q}}} \mathbf{A}_{\mathcal{P} \mathcal{Q}}^{+} \mathbf{Y}_{\mathcal{P}}(\psi) .
\end{aligned}
$$

Where the product $\mathbf{A}_{\mathcal{P} \hat{\mathcal{Q}}} \mathbf{A}_{\mathcal{P} \hat{\mathcal{Q}}}^{+}$is the projection matrix onto the range of $\mathbf{A}_{\mathcal{P} \hat{\mathcal{Q}}}$.

If $\hat{\mathcal{Q}}$ is estimated correctly, the back-projection $\hat{\mathbf{Y}}_{\mathcal{P} \hat{\mathcal{Q}}}(\psi)$ should be approximately equal to $\mathbf{Y}_{\mathcal{P}}(\psi)$, provided the noise level is relatively low. We quantify this through the backprojection error,

$$
\mathrm{BPE}=\int_{0}^{\frac{1}{L}}\left\|\mathbf{Y}_{\mathcal{P}}(\psi)-\hat{\mathbf{Y}}_{\mathcal{P} \hat{\mathcal{Q}}}(\psi)\right\|_{2}^{2} d \psi
$$

\section{A. Sparsity Failure}

Consider for now the case where the noise level is trivially low compared with the received signal power. As discussed in Sec. III, a multi-coset array with a $(P, L)$-universal pattern should be able to recover the support $\mathcal{Q}$ of a $(Q, L)$-sparse scene in most cases given $P \geq Q+1$. When the support estimate is recovered from the response $\mathbf{Y}_{\mathcal{P}}(\psi)=\mathbf{A}_{\mathcal{P} \mathcal{Q}} \mathbf{X}_{\mathcal{Q}}(\psi)$

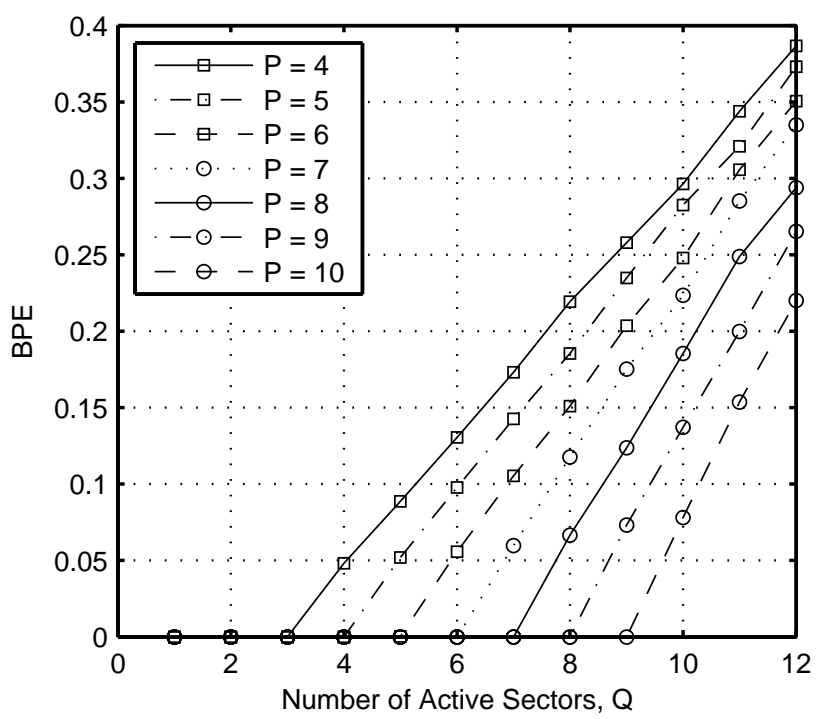

Fig. 6. BPE versus $Q, L=19$. Results averaged over 1000 trials.

is (or contains) the correct support such that $\mathcal{Q} \subseteq \hat{\mathcal{Q}}$, the back-projection is

$$
\begin{aligned}
\hat{\mathbf{Y}}_{\mathcal{P} \hat{\mathcal{Q}}}(\psi) & =\mathbf{A}_{\mathcal{P} \hat{\mathcal{Q}}} \hat{\mathbf{X}}_{\hat{\mathcal{Q}}}(\psi) \\
& =\mathbf{A}_{\mathcal{P} \mathcal{Q}} \mathbf{X}_{\mathcal{Q}}(\psi) \\
& =\mathbf{Y}_{\mathcal{P}}(\psi),
\end{aligned}
$$

and the BPE is zero. If the scene becomes insufficiently sparse for the array, the recovery stage will fail to determine the entirety of the support and $\hat{\mathcal{Q}} \subset \mathcal{Q}$. In this case, much of the energy contained in the unidentified support blocks $\mathcal{Q} / \hat{\mathcal{Q}}$ will vanish during the back-projection operation. These results can be seen in Fig. 6. Each curve represents a fixed number of cosets $P$ for which the average BPE is plotted as a function of the number of supported blocks $Q$. The average BPE was calculated over 1000 trials, each trial having a random gaussian scene evenly distributed over a randomly selected support $\mathcal{Q}$. As expected, each curve remains at zero for $Q<P$ and rises in nearly linear fashion with $Q$ beyond this point.

\section{B. Failure Due to Insufficient SNR}

The BPE behavior with respect to the SNR will behave differently than in the case of false assumptions in the sparsity model. Consider a fixed signal power, distributed over any $Q \leq P-1$ scene blocks. For noise powers below some threshold level (specific to the particular case of $L, Q$, and $\mathcal{P})$, the support recovery will not be adversely affected. In this region, the support $\mathcal{Q}$ will be recovered successfully and the BPE will be due solely to the noise within the subspace orthogonal to the range of $\mathbf{A}_{\mathcal{P Q}}$, which will increase in proportion to the total noise power.

As a consequence, failures occur with increasing likelihood for $P>Q$ at lower SNRs. When operating at a particular SNR, it is possible to determine a "safe" choice of $P$ such that the TSNR for the design is comfortably below this level. However, this may be overly cautious, resulting in the need 


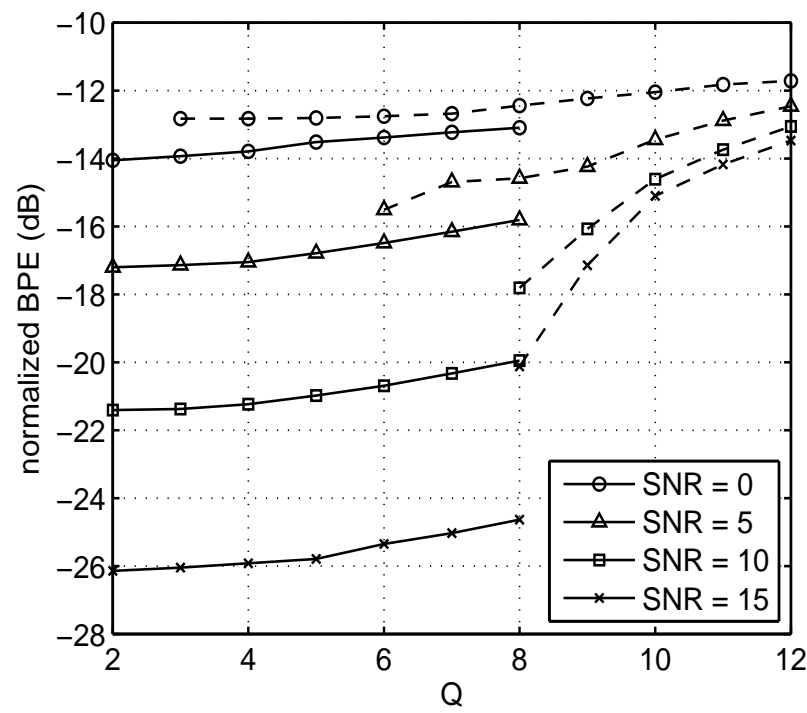

Fig. 7. Normalized BPE versus $Q$, at different SNR, $L=19, P=9$. The solid and dashed portion of each curve represent the successful and failed cases, respectively.

for a much more dense array than necessary. Another strategy is to select an aggressively sparse array design with a TSNR very close to the operating SNR and determine the BPE level that indicates a recovery failure to allow the user to be aware when the relatively rare errors occur. In practice, the BPE threshold at which we declare a failure depends on SNR. This can be seen in Fig. 7, which shows the normalized BPE versus $Q$ for different SNR values for a $(9,19)$ multi-coset array. Rather than averaging the BPE results over every trial as in Fig. 6, the averages are instead taken separately for the cases of successful and failed support recovery estimates. We observe that independent of $Q$, the failed cases consistently lie above some threshold, which varies with SNR. Defining the threshold BPE as the midpoint between the maximum success and minimum failure BPEs allows a nominal level indicating a probable failure to be determined at each SNR. Fig. 8 illustrates this result for the $(9,19)$ array.

\section{RAnge-Angle 2-D Imaging ApPlication}

In this section the multi-coset imaging techniques are applied to create a two-dimensional range-angle image from simulated data. For this, we first discuss the range-dependent sparsity extension which often allows a dense scene to be separated into multiple sparse scenes, each of which may be treated by the imaging algorithm independent of the others.

\section{A. Range-Dependent Sparsity}

The scene sparsity requirements may appear to restrict the potential applications in which a significant reduction in the number of array elements may be achieved. However, even with scenes containing objects in every direction, it is unlikely that many of these objects are located at the same distance from the array. Hence, by sorting the scene into a number of distinct range cells, the multi-coset array can independently

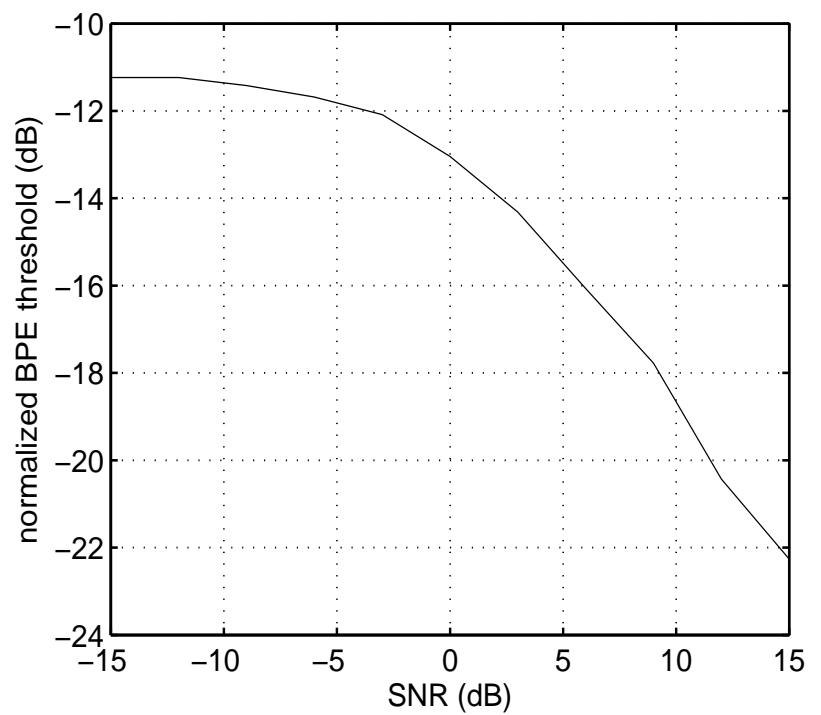

Fig. 8. Normalized BPE threshold versus SNR, $L=19, P=9$.

reconstruct the sparse image in each cell using the standard 1$\mathrm{D}$ imaging algorithm. This notion of range-dependent sparsity can be exploited using standard pulse compression techniques.

When the transmitted waveform contains a range of frequencies $\Delta f$ about the center frequency $f_{0}$, the inverse Fourier transform of the received frequency domain data sorts the response according to the two-way travel times of the various signals reflected from the environment. In a typical medium, each of these signals travel at the same speed, hence sorting by time effectively sorts by distance.

The pulse-compressed range resolution improves linearly with the bandwidth $\Delta f$. As the scene is divided in finer range cells, the resultant range-dependent sparsity profile improves, since the density at any range is monotonically non-increasing as the range cell length $\Delta r$ decreases. The available fractional bandwidth $\Delta f / f_{0}$ of a particular array design is relatively fixed for any $f_{0}$. Hence, exploitation of range-dependent sparsity is inherently well suited for high frequency systems.

\section{B. 2-D Imaging Example}

As a demonstration of multi-coset range-angle imaging, consider the example application of a millimeter-wave vehicular mounted imaging system. Assume a center frequency of $f_{0}=75 \mathrm{GHz}$ and an available aperture length of $2 \mathrm{~m}$. At this frequency, an element spacing of $d=\lambda_{0} / 2=2 \mathrm{~mm}$ implies the need for 1000 array elements in order to fully populate the linear aperture. Further, assume a frequency bandwidth of $1 \mathrm{GHz}$, which provides a $15 \mathrm{~cm}$ range resolution following pulse compression.

The simple line-of-sight point target model shown in Fig. 9 was used to simulate the frequency response at the $N=1000$ array element locations, with the transmitter modeled as an isotropic source located at the center of the array aperture. The full standard array image is generated by first sorting the received data by range using the pulse-compression technique, and then applying (2) at each of the range bins. The result is 


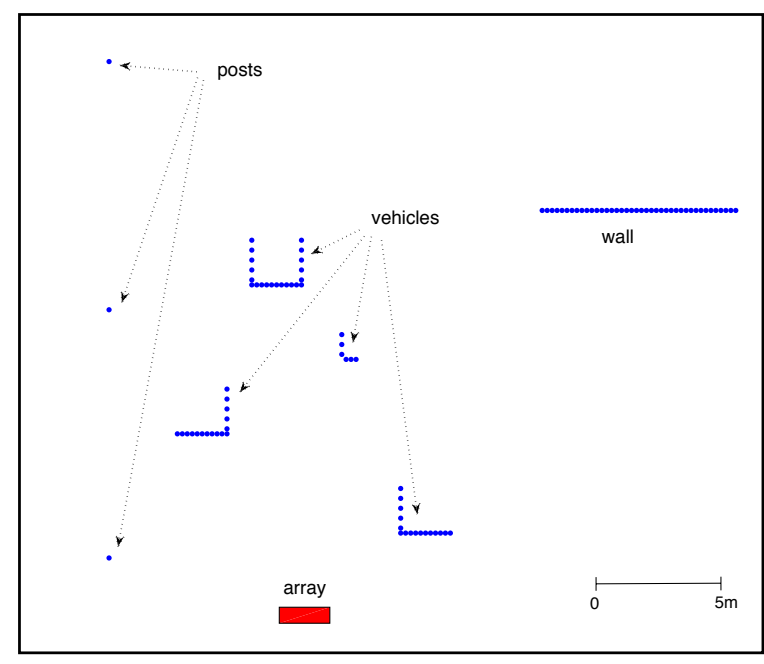

Fig. 9. Point source model.

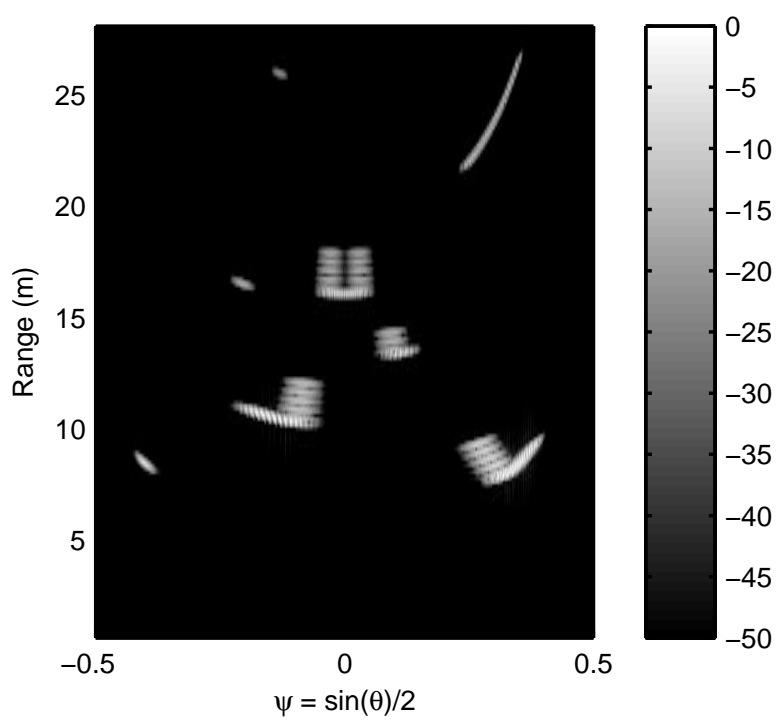

Fig. 10. Standard array image reconstruction, $N=1000$ elements with spacing $d_{0}=\lambda / 2$.

shown in Fig. 10, with the horizontal axis now corresponding to $\psi$-space. Since the block sparsity view divides the scene into sectors of equal widths $\Delta \psi_{L}=1 / L$, this representation governs the number of supported sectors $Q$ for a given $L$.

The significance of the choice of $L$ on the array design can be seen in Fig. 11. The maximum block density $\rho_{s, L}=$ $Q / L$ over all ranges is shown as a function of the number of sectors, and accordingly the coset period. The sparsity of the scene begins to level out around $L=50$ suggesting this as a reasonable choice, with the number of coset periods being $M=N / L=20$.

At $L=50$ the maximum number of occupied blocks is $Q=18$. A conservative pick for the number of cosets is $P=2 Q=36$, resulting in an array with a density factor $\rho_{A}=0.72$. Before we discuss the performance of such an array, it is useful to remind ourselves why designs such as the multi-coset array are needed, rather than simply spacing

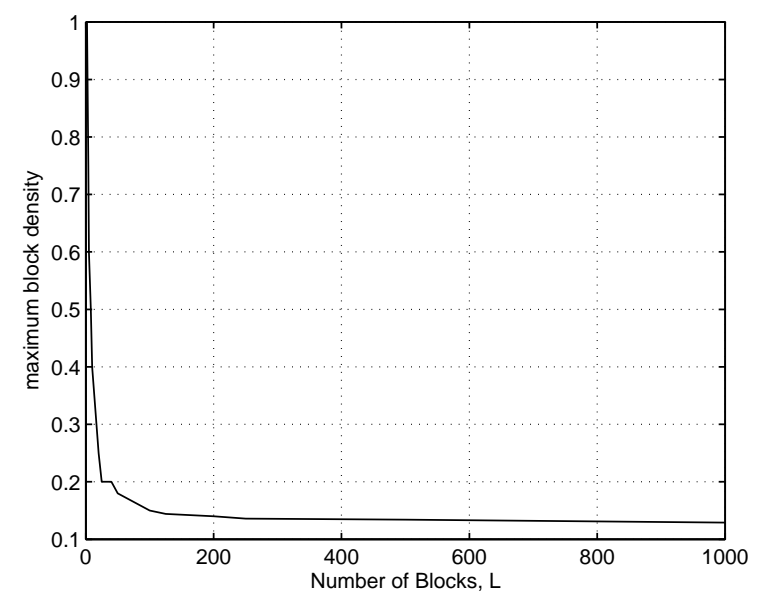

Fig. 11. Maximum block density dependence on the total number of sectors $L$.

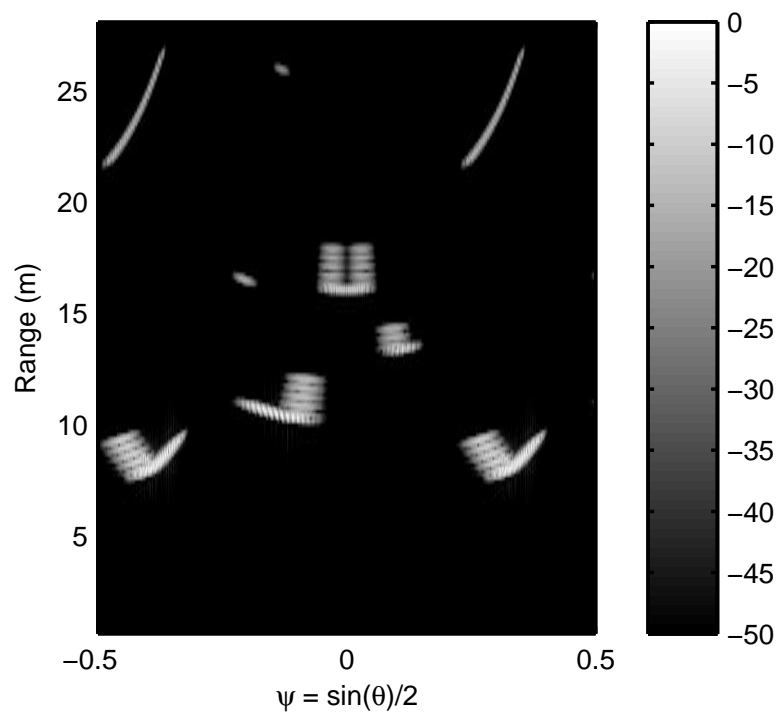

Fig. 12. Reconstructed image from the sparse uniform array of $N=720$ elements with spacing $d=d_{0} / 0.72=0.6944 \lambda$.

the elements a little further than for a standard array. To fill the same aperture with $72 \%$ of the original elements with uniform spacing, the distance between elements will be $d=d_{0} / 0.72=0.6944 \lambda$. Using this configuration to reconstruct the image using the standard imaging approach yields the result shown in Fig. 12. Because of the grating lobe effect, the array is unable to distinguish the direction of arrival for targets outside of $|\psi|<0.36$ and copies of image targets appear in multiple locations, at times even obscuring other objects, as seen at a range of $8 \mathrm{~m}$.

Returning to the multi-coset array, we note that the design procedure laid out in Section IV-C becomes overly cumbersome for large $L$. However, for many cases, a simplified design approach can perform quite well as long as proper care is taken. The desired $(36,50)$ multi-coset array may be designed using a pseudo-random approach by either randomly generating $\mathcal{P}$ and checking to see, for example, that the 


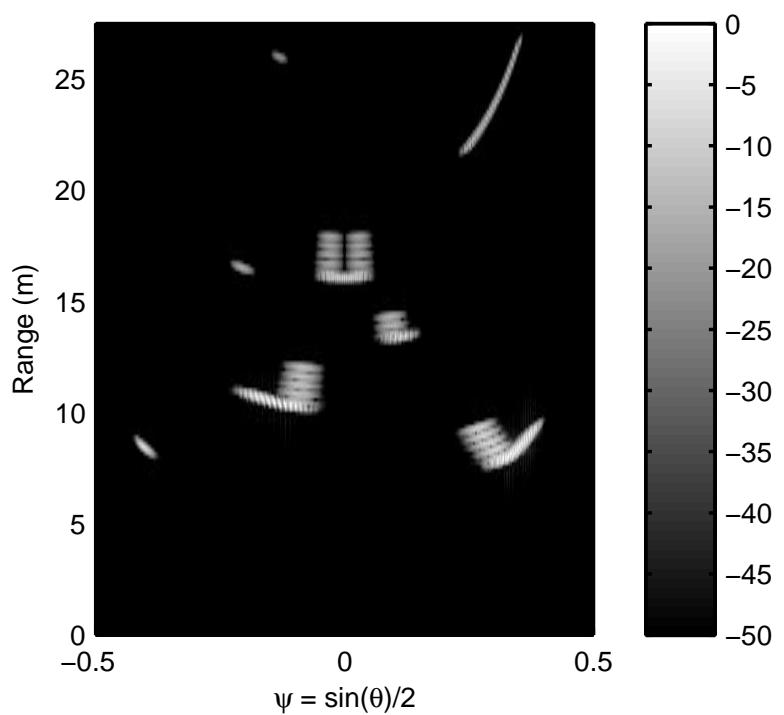

Fig. 13. Reconstructed image for the $(36,50)$ multi-coset array with coset pattern $\mathcal{P}=\{0,2,3,4,6,7,10,11,12,13,14$, $15,16,18,19,20,21,22,24,25,28,29,30,31,32,33,34,35,37,39,40,42$, $44,46,47,48\}$.

elements are not either too tightly bunched or tending to be spread out in a nearly uniform manner, or, by selecting several element locations strategically and then allowing the rest to be selected randomly. The result of a type of this pseudo-random design is shown in Fig. 13. With this conservative choice of $P$, we see that the multi-coset array image reconstruction is nearly indistinguishable from the full array reconstruction.

For this particular case, it is very likely that a randomly selected $\mathcal{P}$ will perform well. However, this does not imply that all patterns will yield good results. Once again, we consider the bunched pattern $\mathcal{P}_{B U}=\{0,1, \ldots, 35\}$ and implement the same multi-coset imaging process, resulting in the odd reconstruction reconstruction result shown in Fig. 14. What we see in this result is the effect of poor conditioning. At ranges for which no actual targets are present, there will always be some presence of very low side lobes resulting from the finite array length and frequency band. While these should be nearly insignificant in most cases, a poorly chosen configuration such as the bunched pattern can amplify trace amounts of background noise by up to several orders of magnitude, as seen in this result.

\section{Range-dependent Failure Detection}

Before we examine the effects of moving to increasingly more sparse arrays, we will apply the failure detection portion of the algorithm to this 2-D imaging process. As was done with the reconstruction algorithm, after separating the coset responses into range bins as described earlier, the failure detection algorithm introduced in Sec. V can be applied to each bin. Fig. 15 shows the reconstructed images for progressively more sparse arrays beginning with $P=Q_{\max }$ and decreasing to $P=Q_{\max } / 3$. The bar to the immediate right indicates the $\mathrm{BPE}$ at each range.

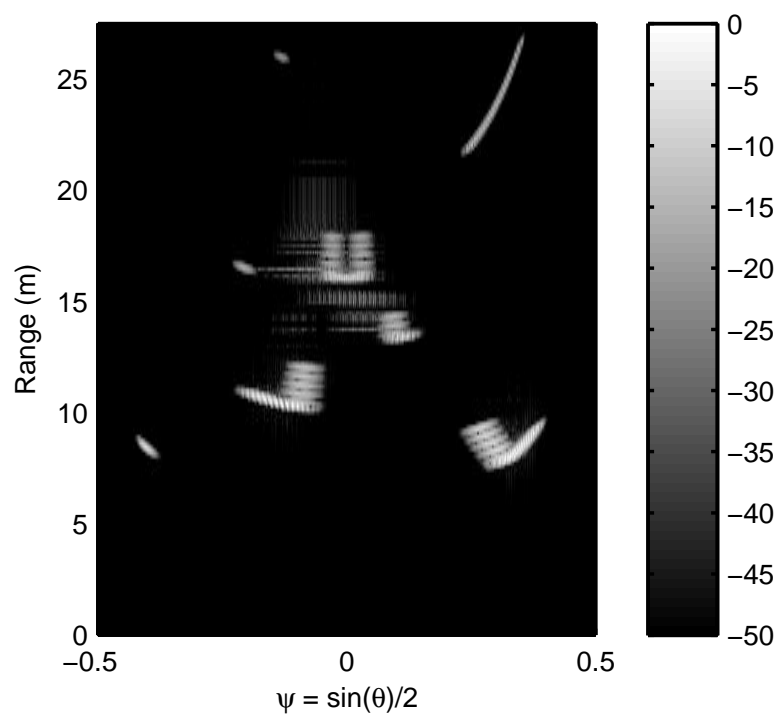

Fig. 14. Reconstructed image for the $(36,50)$ multi-coset array with "bunched" coset pattern $\mathcal{P}_{B U}=\{0,1, \ldots, 35\}$

Due to the moderately aggressive choice of $P=18$ for this scene, the image shown in Fig. 15(a) indicates a mild degree of error at the ranges with the highest densities. This is to be expected as the number of supported blocks is greater than the number of cosets for these cases. This light level of error indication typically corresponds to the case for which the estimated support $\hat{\mathcal{Q}}$ has accurately determined the maximum possible number of supports, with the extra appearing as back projection error. In Fig. 15(b), the array has $P=12$ cosets, and more severe errors begin to occur. A primary utility of having this range-dependent error indication is that when failures occur, the location can be identified and ignored, or judged with caution, without discarding results at other ranges that still have sufficiently low densities. In Fig. 15(c), the array retains $P=9$ cosets, having reduced the total number of elements to 180 of the original $N=1000$. While the objects are showing noticeable levels of distortion, each target is still being located by the support recovery algorithm, with the most egregious corruptions being identified by the error indicator. In Fig. 15(d), $P=6$ cosets remain. At this point, the design procedure must be reconsidered. Counting the number of element pairs as $P(P-1) / 2=15$, this design clearly cannot cover the distribution of pairwise spacing for the $L=50$ coset period. Hence, it makes sense to limit these elements to one side of the period, eliminating the need to consider the wraparound distance. For this case, $\mathcal{P}$ is taken from the 6 element MRLA. While still performing admirably for nearly an order of magnitude reduction in the number of elements, at this level the reconstruction has begun to miss entire objects.

\section{CONCLUDing REMARKS}

We have presented the architecture of the multi-coset sparse imaging array and described the processing algorithm utilized for image reconstruction. While previous sparse array concepts suffered from decreased reconstruction performance, this approach returns images with the same characteristics obtainable 


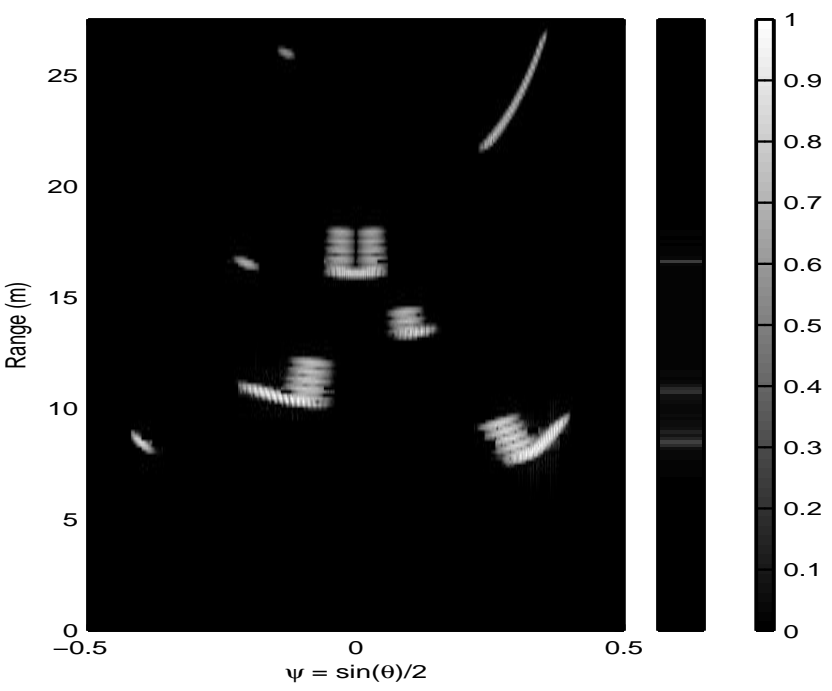

(a)

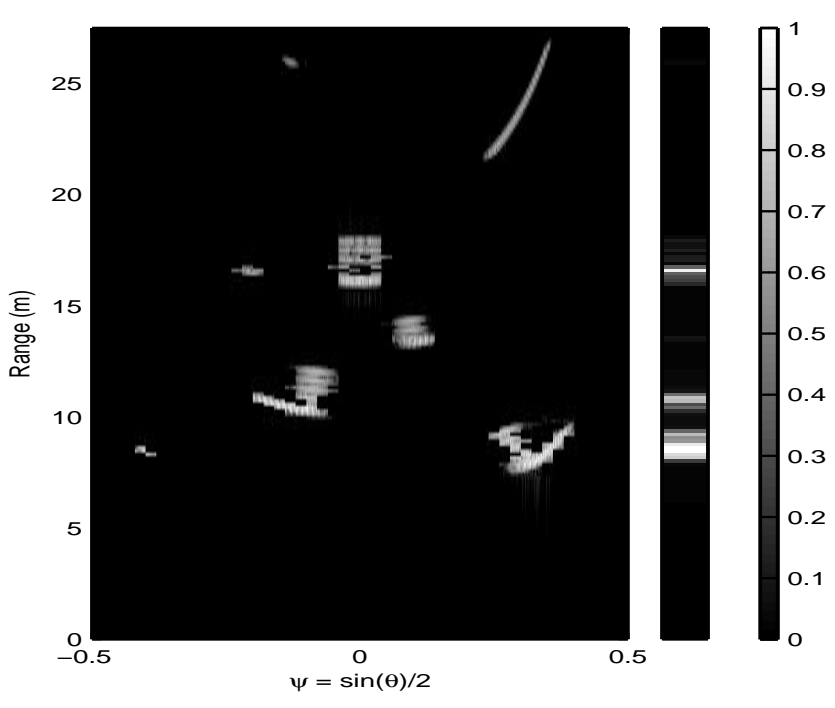

(c)

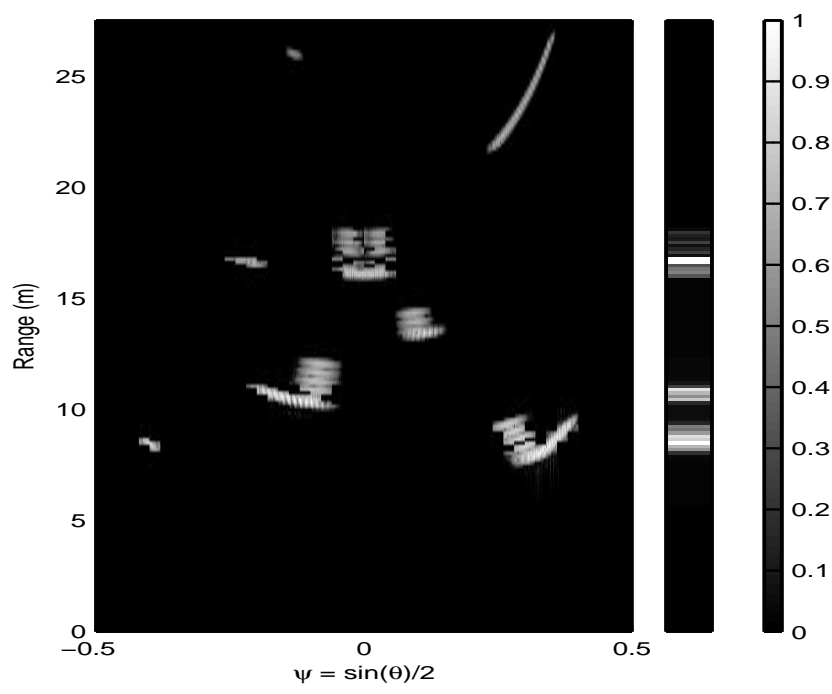

(b)

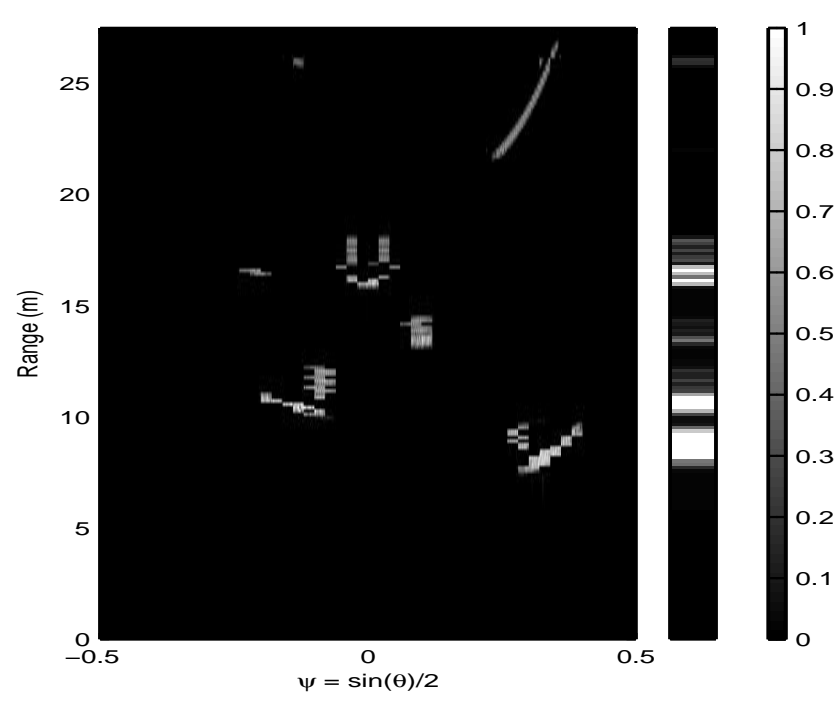

(d)

Fig. 15. Multi-coset images with failure detection, $L=50$ and (a) $P=18$, (b) $P=12$, (c) $P=9$, (d) $P=6$.

with standard arrays. We have demonstrated a modified version of the MUSIC algorithm that yields better low SNR performance than standard versions. A design procedure yielding optimal support recovery performance at low SNR has been detailed. A method for indicating the likelihood of failures has been demonstrated. We also showed how to exploit rangedependent sparsity and create two dimensional range-angle images.

\section{REFERENCES}

[1] Y. T. Lo and S. W. Lee, Antenna Handbook: Antenna Theory. New York, NY: Van Nostrand Reinhold, 1993.

[2] R. O. Schmidt, "Multipe emitter location and signal parameter estimation," IEEE Trans. Antennas Propag., vol. 34, no. 3, pp. 276-280, Mar. 1986.
[3] Y. Kochman and G. W. Wornell, "Finite multi-coset sampling and sparse arrays," in Proc. ITA, La Jolla, CA, 2011, pp. 1-7.

[4] P. Feng and Y. Bresler, "Spectrum-blind minimum-rate sampling and reconstruction of multiband signals," in Proc. ICASSP, vol. 3, Atlanta, GA, 1996, pp. 1688-1691.

[5] Y. M. Lu and M. N. Do, "A theory for sampling signals from a union of subspaces," IEEE Trans. Signal Process., vol. 56, no. 6, pp. 2334-2345, 2008.

[6] J. Chen and X. Huo, "Theoretical results on sparse representations of multiple-measurement vectors," Signal Processing, IEEE Transactions on, vol. 54, no. 12, pp. 4634-4643, 2006.

[7] S. Cotter, B. Rao, K. Engan, and K. Kreutz-Delgado, "Sparse solutions to linear inverse problems with multiple measurement vectors," Signal Processing, IEEE Transactions on, vol. 53, no. 7, pp. 2477-2488, 2005.

[8] M. Mishali and Y. C. Eldar, "Blind multiband signal reconstruction: Compressed sensing for analog signals," IEEE Trans. Signal Process., vol. 57, no. 3, pp. 993-1009, 2009.

[9] G. Bienvenu and L. Kopp, "Optimality of high resolution array processing using the eigensystem approach," IEEE Trans. Acoust., Speech, 
Signal Process., vol. 31, no. 5, pp. 1235-1248, 1983.

[10] H. L. Van Trees, Optimum Array Processing (Detection, Estimation, and Modulation Theory, Part IV). New York, NY: Wiley-Interscience, 2002.

[11] D. Johnson and S. DeGraaf, "Improving the resolution of bearing in passive sonar arrays by eigenvalue analysis," IEEE Trans. Acoust., Speech, Signal Process., vol. 30, no. 4, pp. 638-647, 1982.

[12] A. Moffet, "Minimum-redundancy linear arrays," IEEE Trans. Antennas Propag., vol. 16, no. 2, pp. 172-175, 1968. 